\title{
Estimation of pressure drop in gasket plate heat exchangers
}

\author{
Anisoara Arleziana NEAGU ${ }^{1}$, Claudia Irina $\mathrm{KONCSAG}^{1,{ }^{*}}$, Alina BARBULESCU ${ }^{2}$ and Elisabeta BOTEZ ${ }^{3}$ \\ ${ }^{I}$ Department of Chemistry and Chemical Engineering, "Ovidius" University of Constanta, 124 Mamaia Street, \\ 900521 Constanta, Romania \\ ${ }^{2}$ Higher Colleges of Technology, Sharjah, UAE \\ ${ }^{3}$ Department of Food Science, Food Engineering and Applied Biotechnology, "Dunarea de Jos" University, \\ 111 Domneasca Street, 800201 Galati, Romania
}

\begin{abstract}
In this paper, we present comparatively different methods of pressure drop calculation in the gasket plate heat exchangers (PHEs), using correlations recommended in literature on industrial data collected from a vegetable oil refinery. The goal of this study was to compare the results obtained with these correlations, in order to choose one or two for practical purpose of pumping power calculations. We concluded that pressure drop values calculated with Mulley relationship and Buonopane \& Troupe correlation were close and also Bond's equation gave results pretty close to these but the pressure drop is slightly underestimated. Kumar correlation gave results far from all the others and its application will lead to oversize. In conclusion, for further calculations we will chose either the Mulley relationship or the Buonopane \& Troupe correlation.
\end{abstract}

Keywords: pressure drop, edible oils, gasket plate heat exchangers.

\section{Introduction}

In order to be placed on the market, vegetable oils are subject to a refining process, when the product is improving both in terms of sensorial quality and stability during shelf storage. Gasket plate heat exchangers (PHE) are customary in all modern vegetable oil refineries. The Chevron type gasket plate heat exchangers were introduced in 1930 with dedication to the food industry because they have very good transfer coefficients and can be easily cleaned [1-4].

The calculation of pressure drop is an important part of the technological dimensioning of gasket plate heat exchangers since the power consumption for pumping is determined by the pressure loss in the equipment.

The estimation of pressure drop in exchangers is made with different correlations recommended in literature. All these correlations take into account the geometry of the equipment, the hydrodynamic regime and the physical properties of fluids.

Due to the regular shape of cross-corrugated passages in the PHEs, numerical models can reproduce with fidelity the geometry when investigating the buoyancy effects on pressure losses [5].

In recent years, the authors use finite element computational fluid dynamics (CFD) for the analysis of tortuosity, shape factor and friction factor determination [6].

\footnotetext{
* Corresponding author: ckoncsag@univ-ovidius.ro
}

Also, the Re-Normalization Group (RNG) method is used for the re-normalization of NavierStokes equations in prediction of vortex evolution and the calculation of friction factors and pressure losses [7].

However, different simple correlations developed empirically in laboratory by Kumar, Mulley, Bond, Buonopane, Gulenoglu and other scientists [8-13] can be applied in industrial conditions. The aim of this work was to select a reliable mathematical model from these simple correlations, for the estimation of pressure drop in gasket plate heat exchangers used in the vegetable oil refining industry.

\section{Theoretical approach}

The chevron-type plate is the most used element for PHEs. The plate corrugations are in form of chevron because this pattern is easy to manufacture. Longitudinal and transversal corrugations are plotted in two separated plans [7] and the corrugation angle can be $30^{\circ}, 45^{\circ}$ or $60^{\circ}$, but most frequently $60^{\circ}$.

In Fig. 1, the chevron- type plate is presented with the principal dimensions, some of them being used in hydrodynamic calculations: $\mathrm{L}_{\mathrm{p}}$ - vertical portto-port channel length, $\mathrm{m}$; $\mathrm{L}_{\text {eff- }}$ effective length, $\mathrm{m}$; $\mathrm{L}_{\mathrm{h}}$ - horizontal port-to-port channel length, $\mathrm{m} ; \mathrm{L}_{\mathrm{w}}$ width of flow channel, $m$; $D_{p}$ - port diameter, $m ; \beta$ chevron angle, deg. 


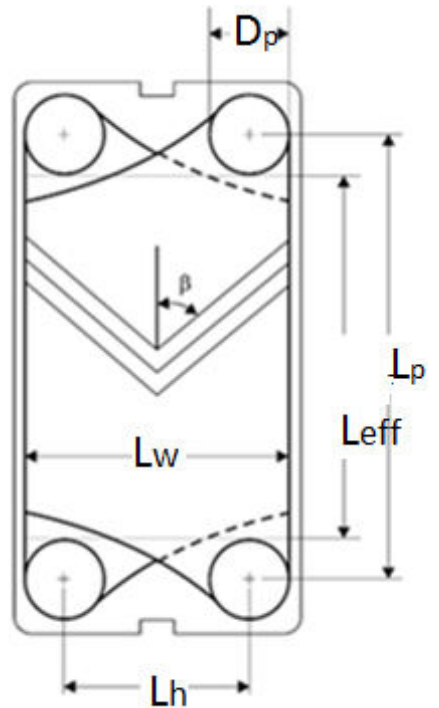

Fig.1.Chevron-type plate

The total pressure drop is the sum of pressure drop in channels and in the ports. It can be estimated with the Eq. (1):

$\Delta p_{t}=\Delta p_{c}+\Delta p_{p}$

where $\Delta p_{t}$ represents the total pressure drop $(\mathrm{Pa})$, $\Delta p_{c}-$ channel pressure drop $(\mathrm{Pa}), \Delta p_{p}$ - port pressure drop $(\mathrm{Pa})$. (2):

The channel pressure drop is defined by the Eq.

$\Delta_{p_{c}}=4 \cdot f \cdot\left(\frac{L_{p} \cdot N_{p}}{d_{h}}\right) \cdot\left(\frac{G_{c h}^{2}}{2 \cdot \rho_{c, h}}\right) \cdot\left(\frac{\mu}{\mu_{w}}\right)^{-0.17}$

where $f$ represents the friction factor, nondimensional; $L_{p}$-vertical port-to-port channel length, $\mathrm{m} ; N_{p}$ - number of passes; $d_{h}$-hydraulic diameter, $\mathrm{m}$; $G_{c h}$ - the fluid mass velocity in the channel, $\mathrm{kg} \cdot \mathrm{m}^{-2} \cdot \mathrm{s}$; $\rho_{c, h}$ - density for the cold respectively for the hot fluid, $\mathrm{kg} / \mathrm{m}^{3} ; \mu-$ dynamic viscosity of fluid at the mean temperature in the apparatus, $\mathrm{Pa} \cdot \mathrm{s} ; \mu_{w}$ dynamic viscosity of fluids at the wall temperature, $\mathrm{Pa} \cdot \mathrm{s}$.

The friction factor $f$ is calculated with different equations from literature as function of Reynolds number and chevron angle.

The pressure drop in the port ducts can be estimated with Eq. (3) [13]:

$\Delta p_{p}=1.4 \cdot N_{p} \cdot\left(\frac{G_{p}^{2}}{2 \cdot \rho_{c, h}}\right)$

where $N_{p}$ represents the number of passes, $G_{p}$-mass velocity of fluid in the port, $\mathrm{kg} \cdot \mathrm{m}^{-2} \cdot \mathrm{s} ; \rho_{c, h}$ - density for cold / hot fluids, $\mathrm{kg} / \mathrm{m}^{3}$.
The mass velocity for the cold and hot fluids in the port is calculated by Eq. (4):

$G_{p}=\frac{\dot{m}}{\pi\left(\frac{D_{p}^{2}}{4}\right)}$

where $\dot{m}$ represents the total flow rate in the port opening $(\mathrm{kg} / \mathrm{s}), D_{p}$ - the port diameter, $\mathrm{m}[6,8,9]$.

As one can see in Eq.1-4, the pressure drop in heat exchangers depends on equipment geometry, physical properties of fluids and flow conditions. The friction factor, $f$, is of great importance and there are dedicated correlations for its estimation.

For example, Kumar correlations (5) and (6) correlate $f$ with the flow regime expressed as Reynolds numbers $[13,14]$ :

$f=\frac{19,400}{R e^{0,589}}$, for $R e=10-100$

$f=\frac{2,990}{R e^{0,183}}$, for $R e>100$

It is important to know that, according to Kumar's observations, the critical Reynolds value for the transition from the laminar to the turbulent flow in PHEs, is approximately $100[6,14]$ since for other authors $[15,16]$, the turbulent regime starts at $\mathrm{Re} \cong$ 400.

The early buoyancy apparition is linked to the shape of cross-corrugated passages and the abrupt change in density and viscosity of fluids due to strong variation of its temperature in a short distance.

Other researchers developed correlations of $f$ with $R e$, in their own way, following experimental studies. All these equations (Eq. 5-10) are empirical: - Bond correlation I [13]:

$f=3,01 \cdot R e^{-0,457}$

- Buonopane \& Troupe correlation [11]:

$f=\frac{2,5}{R e^{0,3}}$

- Bond correlation II [9]:

$f=2,886 \cdot R e^{-0,457}$

- Gulenoglu correlation [12]:

$f=259,9 \cdot R e^{-0,9227}+1,246$

Mulley [12] developed a more complex correlation (Eq. 11) taking into account the most important geometrical dimension, from the viewpoint of friction, the corrugation inclination angle relative to vertical direction, $\beta$, so called chevron angle:

$f=\left(\frac{\beta}{30}\right)^{0,83} \cdot\left[\left(\frac{30,2}{R e}\right)^{5}+\left(\frac{6,28}{R e^{0,5}}\right)^{5}\right]^{0,2}$ 


\section{Experimental}

There were six gasket plate heat exchangers in the industrial plant of different size and with different working fluids (oils with changing properties, cooling water and condensing steam).

In the degumming and neutralization stage, the working fluids are crude oil - bleached oil in PHE \#1, crude oil - steam in PHE \#2, crude oil - water in PHE \#3 and crude oil - steam in PHE \#4. In the winterizing stage, the working fluids are bleached oil - water in PHE \#5 and in the deodorization stage the working fluids are deodorization oil - water in PHE \#6.

The experiment was performed in three campaigns, as follows:

- Campaign 1: processing sunflower oil

- Campaign 2: processing rapeseed oil

- Campaign 3: processing sunflower oil

Every campaign was a few days long, the stock of raw had constant quality during one campaign and the flow rates and temperatures in the process were constant for days. However, inside of each campaign, there were found little changes in flow rates and temperatures, allowing us to have in the end 9 different sets of data for the purposes of this study.

The physical properties of oils (density, viscosity), in all stages of the process, were measured at atmospheric pressure with Anton Parr SVM 3000 apparatus, following the ASTM D445/ISO 121852 method, in the range of $20^{\circ} \mathrm{C}-110^{\circ} \mathrm{C}$, with a precision of $\pm 0,005{ }^{\circ} \mathrm{C}$ for temperature, $\pm 0,0002 \mathrm{~g} / \mathrm{cm}^{3}$ for density and $\pm 0,1 \%$ for the viscosity. The variation curves of density and viscosity with temperature were draw and discussed in previous works [17, 18]. Properties for cooling water and steam were found in [19].

With the geometrical characteristics of each heat exchanger and the physical properties of fluid calculated in the working conditions, the pressure drop was calculated for each circuit with the following correlations: Kumar (Eq. 5 or 6 depending on flow regime), Bond I (Eq. 7), Buonopane \& Troupe (Eq. 8), Bond II (Eq. 9), Gulenoglu (Eq. 10) and Mulley (Eq. 11).

Results are presented in Tables 1-6, each table corresponding to one of the six PHEs in the industrial plant.

\section{Results and Discussions}

For gasket plate heat exchanger PHE \#1, the working fluids are crude oil - bleached oil. Both fluids are liquid and work in the laminar flow $(\operatorname{Re}<100)$. The pressure drop values calculated for this equipment with Eq. 5-11 are presented in Table 1. It can be observed that the highest values of pressure drop are obtained using Kumar relationship Eq. 5, 23 times higher than using other methods. Mulley and Buonopane \& Troupe correlations give closer results, as also seen in Table 7.

Table 1. Pressure drop in PHE \#1 calculated with different correlations

\begin{tabular}{|c|c|c|c|c|c|c|c|c|c|c|}
\hline $\begin{array}{c}\text { Working } \\
\text { fluids }\end{array}$ & $\begin{array}{c}\text { Density, } \\
{\left[\mathbf{k g} / \mathbf{m}^{3}\right]}\end{array}$ & $\begin{array}{c}\text { Dinamic } \\
\text { viscosity, } \\
{[\mathbf{P a} \cdot \mathbf{s}]}\end{array}$ & $\begin{array}{c}\text { Flow } \\
\text { rate, } \\
{[\mathbf{k g} / \mathbf{s}]}\end{array}$ & $\begin{array}{c}\text { Reynolds } \\
\text { number }\end{array}$ & $\begin{array}{c}\text { Pressure } \\
\text { drop, } \\
{[\mathbf{P a}]}\end{array}$ & \multicolumn{2}{c|}{$\begin{array}{c}\text { Kumar } \\
\text { correlation }\end{array}$} & $\begin{array}{c}\text { Mulley } \\
\text { correlation }\end{array}$ & $\begin{array}{c}\text { Bond I } \\
\text { correlation }\end{array}$ & $\begin{array}{c}\text { Buonopane } \\
\text { \& Troupe } \\
\text { correlation }\end{array}$ \\
\hline \multicolumn{2}{|c|}{} \\
\hline
\end{tabular}


A. A. Neagu et al. / Ovidius University Annals of Chemistry 27 (2016) 62-72

\begin{tabular}{|c|c|c|c|c|c|c|c|c|c|}
\hline \multicolumn{10}{|c|}{ Second campaign, rapeseed oil } \\
\hline \multirow{3}{*}{ Crude oil } & \multirow[t]{3}{*}{876.0} & \multirow[t]{3}{*}{0.0162} & \multirow[t]{3}{*}{2.72} & \multirow[t]{3}{*}{45} & $\Delta p_{c}$ & 68356 & 32150 & 17558 & 26562 \\
\hline & & & & & $\Delta p_{p}$ & 9 & 9 & 9 & 9 \\
\hline & & & & & $\Delta p_{t}$ & 68365 & 32159 & 17567 & 26571 \\
\hline \multirow{3}{*}{$\begin{array}{c}\text { Bleached } \\
\text { oil }\end{array}$} & \multirow[t]{3}{*}{888.80} & \multirow[t]{3}{*}{0.0126} & \multirow[t]{3}{*}{3.35} & \multirow[t]{3}{*}{72} & $\Delta p_{c}$ & 83279 & 39870 & 22691 & 36823 \\
\hline & & & & & $\Delta p_{p}$ & 14 & 14 & 14 & 14 \\
\hline & & & & & $\Delta p_{t}$ & 83293 & 39884 & 22705 & 36837 \\
\hline \multicolumn{10}{|c|}{ Third campaign, sunflower oil } \\
\hline \multirow[t]{3}{*}{ Crude oil } & \multirow[t]{3}{*}{889.9} & \multirow[t]{3}{*}{0.0142} & \multirow[t]{3}{*}{1.736} & \multirow[t]{3}{*}{33} & $\Delta p_{c}$ & 33464 & 15837 & 8243 & 11864 \\
\hline & & & & & $\Delta p_{p}$ & 4 & 4 & 4 & 4 \\
\hline & & & & & $\Delta p_{t}$ & 33468 & 15841 & 8247 & 11868 \\
\hline \multirow{3}{*}{$\begin{array}{c}\text { Bleached } \\
\text { oil }\end{array}$} & \multirow[t]{3}{*}{877.1} & \multirow[t]{3}{*}{0.011} & \multirow[t]{3}{*}{2.138} & \multirow[t]{3}{*}{53} & $\Delta p_{c}$ & 41509 & 19587 & 10870 & 16827 \\
\hline & & & & & $\Delta p_{p}$ & 6 & 6 & 6 & 6 \\
\hline & & & & & $\Delta p_{t}$ & 41515 & 19593 & 10876 & 16833 \\
\hline \multirow[t]{3}{*}{ Crude oil } & \multirow[t]{3}{*}{889.9} & \multirow[t]{3}{*}{0.0142} & \multirow[t]{3}{*}{2.049} & \multirow[t]{3}{*}{39} & $\Delta p_{c}$ & 42283 & 19893 & 10646 & 15726 \\
\hline & & & & & $\Delta p_{r}$ & 5 & 5 & 5 & 5 \\
\hline & & & & & $\Delta p_{t}$ & 42288 & 19898 & 10651 & 15731 \\
\hline \multirow{3}{*}{$\begin{array}{c}\text { Bleached } \\
\text { oil }\end{array}$} & 877.1 & 0.011 & 2.524 & 62 & $\Delta p_{c}$ & 5244 & 24921 & 14038 & 22304 \\
\hline & & & & & $\Delta p_{p}$ & 8 & 8 & 8 & 8 \\
\hline & & & & & $\Delta p_{t}$ & 52455 & 24929 & 14046 & 22312 \\
\hline Crude oil & 889.9 & 0.0142 & 2.457 & 47 & $\Delta p_{c}$ & 54631 & 25706 & 14088 & 21414 \\
\hline & & & & & $\Delta p_{p}$ & 8 & 8 & 8 & 8 \\
\hline & & & & & $\Delta p_{t}$ & 54639 & 25713 & 14096 & 21422 \\
\hline Bleached & 877.1 & 0.011 & 3.026 & 75 & $\Delta p_{c}$ & 67764 & 32537 & 18578 & 30371 \\
\hline oil & & & & & $\Delta p_{r}$ & 12 & 12 & 12 & 12 \\
\hline & & & & & $\Delta p_{t}$ & 67776 & 32549 & 18590 & 30383 \\
\hline Crude oil & 889.9 & 0.0142 & 2.713 & 52 & $\Delta p_{c}$ & 62832 & 29633 & 16416 & 25344 \\
\hline & & & & & $\Delta p_{r}$ & 9 & 9 & 9 & 9 \\
\hline & & & & & $\Delta p_{t}$ & 62841 & 29642 & 16426 & 25353 \\
\hline Bleached & 877.1 & 0.011 & 3.342 & 82 & $\Delta p_{c}$ & 77935 & 37668 & 21648 & 35945 \\
\hline oil & & & & & $\Delta p_{r}$ & 14 & 14 & 14 & 14 \\
\hline & & & & & $\Delta p_{t}$ & 77494 & 37682 & 21662 & 35959 \\
\hline
\end{tabular}

The working fluids for gasket plate heat exchanger PHE \#2 are crude oil and steam. The results of pressure drop for crude oil - steam are presented in Table 2. Reynolds numbers indicate a turbulent flow either we accept the critical Re $>100[6$, 14], or $\operatorname{Re}>400[15,16]$ for the transition to the turbulent flow. As a consequences, pressure drop on the oil circuit are higher than those in the PDE \#1.

It can be observed from Table 2 that the Kumar relationship give the highest values of pressure drops for both oil and steam circuits. The pressure drop on the condensing steam circuit is higher than on oil because the turbulent flow is well developed. By comparing the values of pressure drop in the steam circuit, there are observed high differences, the Kumar correlation giving results tenfold higher than Bond correlation and even differences between the other correlations' results are important. This is due to the fact that all these correlations were produced for liquids working in PHEs.

Table 2. Pressure drop values in PHE \#2 calculated with different correlations

\begin{tabular}{|c|c|c|c|c|c|c|c|c|c|}
\hline $\begin{array}{l}\text { Working } \\
\text { fluids }\end{array}$ & $\begin{array}{l}\text { Density, } \\
{\left[\mathrm{kg} / \mathrm{m}^{3}\right]}\end{array}$ & $\begin{array}{c}\text { Dinamic } \\
\text { viscosity, } \\
{[\mathrm{Pa} \cdot \mathbf{s}]}\end{array}$ & $\begin{array}{l}\text { Flow } \\
\text { rate, } \\
{[\mathrm{kg} / \mathrm{s}]}\end{array}$ & $\begin{array}{c}\text { Pressure } \\
\text { drop, } \\
\text { [Pa] }\end{array}$ & $\begin{array}{c}\text { Reynolds } \\
\text { number }\end{array}$ & $\begin{array}{c}\text { Kumar } \\
\text { correlation }\end{array}$ & $\begin{array}{c}\text { Mulley } \\
\text { correlation }\end{array}$ & $\begin{array}{l}\text { Buonopane } \\
\text { \& Troupe } \\
\text { correlation }\end{array}$ & $\begin{array}{c}\text { Bond I } \\
\text { correlation }\end{array}$ \\
\hline \multicolumn{10}{|c|}{ First campaign, sunflower oil } \\
\hline \multirow[t]{3}{*}{ Crude oil } & \multirow[t]{3}{*}{874.3} & \multirow[t]{3}{*}{0.0087} & \multirow[t]{3}{*}{1.736} & $\Delta p_{c}$ & \multirow[t]{3}{*}{328} & 50223 & 39621 & 24339 & 11830 \\
\hline & & & & $\Delta p_{p}$ & & 3 & 3 & 3 & 3 \\
\hline & & & & $\Delta p_{t}$ & & 50227 & 39624 & 24342 & 11833 \\
\hline \multirow[t]{3}{*}{ Steam } & \multirow[t]{3}{*}{1.923} & \multirow[t]{3}{*}{0.000015} & \multirow[t]{3}{*}{0.023} & $\Delta p_{c}$ & \multirow[t]{3}{*}{9309} & 121950 & 14136 & 35002 & 10037 \\
\hline & & & & $\Delta p_{p}$ & & 6 & 6 & 6 & 6 \\
\hline & & & & $\Delta p_{t}$ & & 121956 & 14142 & 35008 & 10042 \\
\hline \multirow[t]{3}{*}{ Crude oil } & \multirow[t]{3}{*}{874.3} & \multirow[t]{3}{*}{0.0087} & \multirow[t]{3}{*}{2.049} & $\Delta p_{c}$ & \multirow[t]{3}{*}{387} & 66606 & 46261 & 31488 & 15999 \\
\hline & & & & $\Delta p_{p}$ & & 5 & 5 & 5 & 5 \\
\hline & & & & $\Delta p_{t}$ & & 666611 & 46266 & 31493 & 16004 \\
\hline \multirow[t]{2}{*}{ Steam } & \multirow[t]{2}{*}{1.923} & \multirow[t]{2}{*}{0.000015} & \multirow[t]{2}{*}{0.028} & $\Delta p_{c}$ & \multirow[t]{2}{*}{10987} & 164814 & 18135 & 46396 & 12962 \\
\hline & & & & $\Delta p_{p}$ & & 8 & 8 & 8 & 8 \\
\hline
\end{tabular}


A. A. Neagu et al. / Ovidius University Annals of Chemistry 27 (2016) 62-72

\begin{tabular}{|c|c|c|c|c|c|c|c|c|c|}
\hline & & & & $\Delta p_{t}$ & & 164822 & 18143 & 46396 & 12970 \\
\hline \multirow[t]{3}{*}{ Crude oil } & \multirow[t]{3}{*}{874.3} & \multirow[t]{3}{*}{0.0087} & \multirow[t]{3}{*}{2.475} & $\Delta p_{c}$ & \multirow[t]{3}{*}{467} & 91885 & 50814 & 42167 & 22536 \\
\hline & & & & $\Delta p_{p}$ & & 7 & 7 & 7 & 7 \\
\hline & & & & $\Delta p_{t}$ & & 91892 & 50821 & 42174 & 22542 \\
\hline \multirow[t]{3}{*}{ Steam } & \multirow[t]{3}{*}{1.923} & \multirow[t]{3}{*}{0.000015} & \multirow[t]{3}{*}{0.033} & $\Delta p_{c}$ & \multirow[t]{3}{*}{13175} & 229239 & 23802 & 63175 & 17155 \\
\hline & & & & $\Delta p_{p}$ & & 11 & 11 & 11 & 11 \\
\hline & & & & $\Delta p_{t}$ & & 229250 & 23813 & 63186 & 17156 \\
\hline \multirow[t]{3}{*}{ Crude oil } & \multirow[t]{3}{*}{874.3} & \multirow[t]{3}{*}{0.0087} & \multirow[t]{3}{*}{2.713} & $\Delta p_{c}$ & \multirow[t]{3}{*}{513} & 107664 & 77655 & 48709 & 26734 \\
\hline & & & & $\Delta p_{p}$ & & 8 & 8 & 8 & 8 \\
\hline & & & & $\Delta p_{t}$ & & 107671 & 77663 & 48717 & 26743 \\
\hline \multirow[t]{3}{*}{ Steam } & \multirow[t]{3}{*}{1.923} & \multirow[t]{3}{*}{0.000015} & \multirow[t]{3}{*}{0.037} & $\Delta p_{c}$ & \multirow[t]{3}{*}{14548} & 274474 & 27618 & 74769 & 19989 \\
\hline & & & & $\Delta p_{p}$ & & 14 & 14 & 14 & 14 \\
\hline & & & & $\Delta p_{t}$ & & 274488 & 27632 & 74783 & 20003 \\
\hline \multicolumn{10}{|c|}{ Second campaign, rapeseed oil } \\
\hline \multirow[t]{3}{*}{ Crude oil } & 873.0 & 0.011 & 2.72 & $\Delta p_{c}$ & 425 & 18583 & 93262 & 57976 & 18531 \\
\hline & & & & $\Delta p_{p}$ & & 36 & 36 & 36 & 36 \\
\hline & & & & $\Delta p_{t}$ & & 18619 & 93270 & 57985 & 18567 \\
\hline Steam & 1.923 & 0.000015 & 0.037 & $\Delta p_{c}$ & 14585 & 274625 & 27819 & 74992 & 20105 \\
\hline & & & & $\Delta p_{p}$ & & 15 & 15 & 15 & 15 \\
\hline & & & & $\Delta p_{t}$ & & 274640 & 27834 & 75007 & 20120 \\
\hline & & & & Third & aign, s & wer oil & & & \\
\hline Crude oil & 873.2 & 0.0091 & 1.736 & $\Delta p_{c}$ & 313 & 58810 & 43562 & 26925 & 22612 \\
\hline & & & & $\Delta p_{p}$ & & 4 & 4 & 4 & 4 \\
\hline & & & & $\Delta p_{t}$ & & 58814 & 43562 & 26929 & 22616 \\
\hline Steam & 1.923 & 0.000015 & 0.028 & $\Delta p_{c}$ & 9309 & 121950 & 14136 & 35002 & 10037 \\
\hline & & & & $\Delta p_{p}$ & & 6 & 6 & 6 & 6 \\
\hline & & & & $\Delta p_{t}$ & & 121956 & 14142 & 35008 & 10042 \\
\hline Crude oil & 873.2 & 0.0091 & 2.049 & $\Delta p_{c}$ & 369 & 73513 & 56631 & 34991 & 28251 \\
\hline & & & & $\Delta p_{p}$ & & 5 & 5 & 5 & 5 \\
\hline & & & & $\Delta p_{t}$ & & 73518 & 56636 & 34996 & 28256 \\
\hline Steam & 1.923 & 0.000015 & 0.033 & $\Delta p_{c}$ & 10987 & 164814 & 18135 & 46396 & 12962 \\
\hline & & & & $\Delta p_{p}$ & & 8 & 8 & 8 & 8 \\
\hline & & & & $\Delta p_{t}$ & & 164822 & 18143 & 46396 & 12970 \\
\hline Crude oil & 873.2 & 0.0091 & 2.475 & $\Delta p_{c}$ & 443 & 100100 & 74357 & 46307 & 39294 \\
\hline & & & & $\Delta p_{r}$ & & 7 & 7 & 7 & 7 \\
\hline & & & & $\Delta p_{t}$ & & 100107 & 74363 & 46314 & 39301 \\
\hline Steam & 1.923 & 0.000015 & 0.033 & $\Delta p_{c}$ & 13175 & 229239 & 23802 & 63175 & 17155 \\
\hline & & & & $\Delta p_{p}$ & & 11 & 11 & 11 & 11 \\
\hline & & & & $\Delta p_{t}$ & & 229250 & 23813 & 63186 & 17156 \\
\hline Crude oil & 873.2 & 0.0091 & 2.713 & $\Delta p_{c}$ & 489 & 118470 & 86273 & 53959 & 47047 \\
\hline & & & & $\Delta p_{p}$ & & 8 & 8 & 8 & 8 \\
\hline & & & & $\Delta p_{t}$ & & 118478 & 86281 & 53968 & 47052 \\
\hline Steam & 1.923 & 0.000015 & 0.037 & $\Delta p_{c}$ & 14548 & 274474 & 27618 & 74769 & 19989 \\
\hline & & & & $\Delta p_{p}$ & & 14 & 14 & 14 & 14 \\
\hline & & & & $\Delta p_{t}$ & & 274488 & 27632 & 74783 & 20003 \\
\hline
\end{tabular}

Working fluids for gasket plate heat exchanger PHE\#3 are crude oil and water. Table 3 presents the values of pressure drops.

From Table 3, also it can be observed that highest values of pressure drop are obtained with Kumar correlation. Also, there are higher values of pressure drop on water circuit because the mass velocity of water is three times higher than that of oil in similar flow sections; it is to be considered that pressure drop is also dependent on friction factor, $f$, decreasing with $\mathrm{Re}$, and $f$ is four times higher in the oil circuit. The combined effect of this two antagonist factors led to pressure drop double in the water circuit.

For gasket plate heat exchanger PHE \#4, the working fluids are crude oil and steam. The pressure drop values for this equipment are presented in Table 4. 
A. A. Neagu et al. / Ovidius University Annals of Chemistry 27 (2016) 62-72

Table 3. Pressure drop values in PHE \#3 calculated with different correlations

\begin{tabular}{|c|c|c|c|c|c|c|c|c|c|}
\hline $\begin{array}{l}\text { Working } \\
\text { fluids }\end{array}$ & $\begin{array}{l}\text { Density, } \\
{\left[\mathrm{kg} / \mathrm{m}^{3}\right]}\end{array}$ & $\begin{array}{c}\text { Dinamic } \\
\text { viscosity, } \\
{[\mathrm{Pa} \cdot \mathrm{s}]}\end{array}$ & $\begin{array}{l}\text { Flow } \\
\text { rate, } \\
{[\mathrm{kg} / \mathrm{s}]}\end{array}$ & $\begin{array}{c}\text { Pressure } \\
\text { drop, } \\
\text { [Pa] }\end{array}$ & $\begin{array}{c}\text { Reynolds } \\
\text { number }\end{array}$ & $\begin{array}{c}\text { Kumar } \\
\text { correlation }\end{array}$ & $\begin{array}{c}\text { Mulley } \\
\text { correlation }\end{array}$ & $\begin{array}{c}\text { Bond I } \\
\text { correlation }\end{array}$ & $\begin{array}{c}\text { Buonopane } \\
\text { \& Troupe } \\
\text { correlation }\end{array}$ \\
\hline \multicolumn{10}{|c|}{ First campaign, sunflower oil } \\
\hline \multirow[t]{3}{*}{ Crude oil } & \multirow[t]{3}{*}{888.0} & \multirow[t]{3}{*}{0.0157} & \multirow[t]{3}{*}{1.736} & $\Delta p_{c}$ & \multirow[t]{3}{*}{25} & 13563 & 11185 & 3212 & 4415 \\
\hline & & & & $\Delta p_{p}$ & & 2 & 2 & 2 & 2 \\
\hline & & & & $\Delta p_{t}$ & & 13565 & 11187 & 3214 & 4417 \\
\hline \multirow[t]{3}{*}{ Water } & \multirow[t]{3}{*}{987.9} & \multirow[t]{3}{*}{0.00077} & \multirow[t]{3}{*}{5.251} & $\Delta p_{c}$ & \multirow[t]{3}{*}{154} & 26009 & 6978 & 5135 & 9203 \\
\hline & & & & $\Delta p_{p}$ & & 15 & 15 & 15 & 15 \\
\hline & & & & $\Delta p_{t}$ & & 26024 & 6993 & 5150 & 9218 \\
\hline \multirow[t]{3}{*}{ Crude oil } & \multirow[t]{3}{*}{888.0} & \multirow[t]{3}{*}{0.0157} & \multirow[t]{3}{*}{2.049} & $\Delta p_{c}$ & 29 & 17136 & 14570 & 4149 & 5852 \\
\hline & & & & $\Delta p_{p}$ & & 3 & 3 & 3 & 3 \\
\hline & & & & $\Delta p_{t}$ & & 17139 & 14572 & 4152 & 5855 \\
\hline Water & 987.9 & 0.00077 & 6.198 & $\Delta p_{c}$ & 1782 & 33701 & 8134 & 6616 & 11766 \\
\hline & & & & $\Delta p_{p}$ & & 21 & 21 & 21 & 21 \\
\hline & & & & $\Delta p_{t}$ & & 33723 & 8156 & 6637 & 11788 \\
\hline Crude oil & 888.0 & 0.0157 & 2.475 & $\Delta p_{c}$ & 35 & 22142 & 19634 & 5491 & 7969 \\
\hline & & & & $\Delta p_{p}$ & & 4 & 4 & 4 & 4 \\
\hline & & & & $\Delta p_{t}$ & & 22146 & 19638 & 5495 & 7973 \\
\hline Water & 987.9 & 0.00077 & 7.432 & $\Delta p_{c}$ & 2155 & 47596 & 11397 & 9272 & 16211 \\
\hline & & & & $\Delta p_{p}$ & & 31 & 31 & 31 & 31 \\
\hline & & & & $\Delta p_{t}$ & & 47627 & 11428 & 9303 & 16242 \\
\hline Crude oil & 888.0 & 0.0157 & 2.713 & $\Delta p_{c}$ & 39 & 25476 & 23182 & 6402 & 9437 \\
\hline & & & & $\Delta p_{p}$ & & 5 & 5 & 5 & 5 \\
\hline & & & & $\Delta p_{t}$ & & 25480 & 23186 & 6407 & 9441 \\
\hline Water & 987.9 & 0.00077 & 8.206 & $\Delta p_{c}$ & 2386 & 57321 & 13701 & 15126 & 19266 \\
\hline & & & & $\Delta p_{p}$ & & 38 & 38 & 38 & 38 \\
\hline & & & & $\Delta p_{t}$ & & 57359 & 13740 & 15164 & 19304 \\
\hline & & & & Second & mpaign, ra & seed oil & & & \\
\hline Crude oil & 886.7 & 0.0152 & 2.720 & $\Delta p_{c}$ & 40 & 24608 & 22824 & 6211 & 9201 \\
\hline & & & & $\Delta p_{p}$ & & 5 & 5 & 5 & 5 \\
\hline & & & & $\Delta p_{t}$ & & 24612 & 22829 & 6215 & 9205 \\
\hline Water & 987.9 & 0.00077 & 8.227 & $\Delta p_{c}$ & 2391 & 57511 & 13769 & 11194 & 19352 \\
\hline & & & & $\Delta p_{p}$ & & 38 & 38 & 38 & 38 \\
\hline & & & & $\Delta p_{t}$ & & 57549 & 13807 & 11232 & 19390 \\
\hline & & & & Third c & ipaign, sun & wer oil & & & \\
\hline Crude oil & 886.9 & 0.0132 & 1.736 & $\Delta p_{c}$ & 29 & 12034 & 11439 & 2915 & 4116 \\
\hline & & & & $\Delta p_{p}$ & & 2 & 2 & 2 & 2 \\
\hline & & & & $\Delta p_{t}$ & & 12036 & 11441 & 2917 & 4118 \\
\hline Water & 987.9 & 0.00077 & 5.251 & $\Delta p_{c}$ & 1526 & 25433 & 6942 & 5017 & 9018 \\
\hline & & & & $\Delta p_{p}$ & & 16 & 16 & 16 & 16 \\
\hline & & & & $\Delta p_{t}$ & & 25449 & 6958 & 5033 & 9034 \\
\hline Crude oil & 886.9 & 0.0132 & 2.049 & $\Delta p_{c}$ & 35 & 15207 & 15586 & 3765 & 5457 \\
\hline & & & & $\Delta p_{p}$ & & 3 & 3 & 3 & 3 \\
\hline & & & & $\Delta p_{t}$ & & 15210 & 15589 & 3788 & 5460 \\
\hline Water & 987.9 & 0.00077 & 6.198 & $\Delta p_{c}$ & 1801 & 34372 & 7230 & 6747 & 11955 \\
\hline & & & & $\Delta p_{p}$ & & 22 & 22 & 22 & 22 \\
\hline & & & & $\Delta p_{t}$ & & 34394 & 7252 & 6769 & 11977 \\
\hline Crude oil & 886.9 & 0.0132 & 2.475 & $\Delta p_{c}$ & 42 & 19648 & 17751 & 4984 & 7430 \\
\hline & & & & $\Delta p_{p}$ & & 4 & 4 & 4 & 4 \\
\hline & & & & $\Delta p_{t}$ & & 19651 & 17755 & 4988 & 7434 \\
\hline Water & 987.9 & 0.00077 & 7.432 & $\Delta p_{c}$ & 2160 & 47808 & 11285 & 9334 & 16279 \\
\hline & & & & $\Delta p_{p}$ & & 31 & 31 & 31 & 31 \\
\hline & & & & $\Delta p_{t}$ & & 47839 & 11316 & 9365 & 16310 \\
\hline Crude oil & 886.9 & 0.0132 & 2.713 & $\Delta p_{c}$ & 46 & 22597 & 20794 & 5842 & 8794 \\
\hline & & & & $\Delta p_{p}$ & & 5 & 5 & 5 & 5 \\
\hline & & & & $\Delta p_{t}$ & & 22602 & 20799 & 5847 & 8799 \\
\hline Water & 987.9 & 0.00077 & 8.206 & $\Delta p_{c}$ & 2385 & 5724 & 13584 & 1143 & 19267 \\
\hline & & & & $\Delta p_{p}$ & & 38 & 38 & 38 & 38 \\
\hline & & & & $\Delta p_{t}$ & & 57280 & 13622 & 11181 & 19305 \\
\hline
\end{tabular}


Table 4. Pressure drop values in PHE \#4 calculated with different correlations

\begin{tabular}{|c|c|c|c|c|c|c|c|c|c|}
\hline $\begin{array}{l}\text { Working } \\
\text { fluids }\end{array}$ & $\begin{array}{c}\text { Density, } \\
{\left[\mathrm{kg} / \mathrm{m}^{3}\right]}\end{array}$ & $\begin{array}{c}\text { Dinamic } \\
\text { viscosity, } \\
{[\mathrm{Pa} \cdot \mathrm{s}]}\end{array}$ & $\begin{array}{l}\text { Flow } \\
\text { rate, } \\
{[\mathrm{kg} / \mathrm{s}]}\end{array}$ & $\begin{array}{c}\text { Pressure } \\
\text { drop, }[\mathrm{Pa}]\end{array}$ & $\begin{array}{c}\text { Reynolds } \\
\text { number }\end{array}$ & $\begin{array}{c}\text { Kumar } \\
\text { correlation }\end{array}$ & $\begin{array}{c}\text { Mulley } \\
\text { correlation }\end{array}$ & $\begin{array}{c}\text { Bond I } \\
\text { correlation }\end{array}$ & $\begin{array}{c}\text { Buonopane } \\
\text { \& Troupe } \\
\text { correlation }\end{array}$ \\
\hline \multicolumn{10}{|c|}{ First campaign, sunflower oil } \\
\hline \multirow[t]{3}{*}{ Crude oil } & \multirow[t]{3}{*}{883.4} & \multirow[t]{3}{*}{0.0128} & \multirow[t]{3}{*}{1.736} & $\Delta p_{c}$ & \multirow[t]{3}{*}{102} & 33443 & 16312 & 9495 & 16278 \\
\hline & & & & $\Delta p_{r}$ & & 14 & 14 & 14 & 14 \\
\hline & & & & $\Delta p_{t}$ & & 33457 & 16326 & 9519 & 16292 \\
\hline \multirow[t]{3}{*}{ Steam } & \multirow[t]{3}{*}{1.923} & \multirow[t]{3}{*}{0.000015} & \multirow[t]{3}{*}{0.050} & $\Delta p_{c}$ & \multirow[t]{3}{*}{9104} & 89006 & 10390 & 7370 & 25613 \\
\hline & & & & $\Delta p_{r}$ & & 73 & 73 & 73 & 73 \\
\hline & & & & $\Delta p_{t}$ & & 89079 & 10443 & 7443 & 25686 \\
\hline \multirow[t]{3}{*}{ Crude oil } & \multirow[t]{3}{*}{883.4} & \multirow[t]{3}{*}{0.0128} & \multirow[t]{3}{*}{2.049} & $\Delta p_{c}$ & 120 & 45131 & 20840 & 12230 & 21532 \\
\hline & & & & $\Delta p_{r}$ & & 19 & 19 & 19 & 19 \\
\hline & & & & $\Delta p_{t}$ & & 45150 & 20859 & 12249 & 21551 \\
\hline Steam & 1.923 & 0.000015 & 0.059 & $\Delta p_{c}$ & 10745 & 120291 & 13323 & 9519 & 33950 \\
\hline & & & & $\Delta p_{r}$ & & 101 & 101 & 101 & 101 \\
\hline & & & & $\Delta p_{t}$ & & 120392 & 13424 & 9620 & 34051 \\
\hline Crude oil & 883.4 & 0.0128 & 2.475 & $\Delta p_{c}$ & 144 & 63085 & 27440 & 16242 & 29499 \\
\hline & & & & $\Delta p_{r}$ & & 28 & 28 & 28 & 28 \\
\hline & & & & $\Delta p_{t}$ & & 63113 & 27468 & 16270 & 29527 \\
\hline Steam & 1.923 & 0.000015 & 0.071 & $\Delta p_{c}$ & 12885 & 167311 & 17495 & 12597 & 46230 \\
\hline & & & & $\Delta p_{r}$ & & 145 & 145 & 145 & 145 \\
\hline & & & & $\Delta p_{t}$ & & 167456 & 17640 & 12742 & 46375 \\
\hline Crude oil & 883.4 & 0.0128 & 2.713 & $\Delta p_{c}$ & 159 & 75320 & 31778 & 18912 & 34792 \\
\hline & & & & $\Delta p_{r}$ & & 35 & 35 & 35 & 35 \\
\hline & & & & $\Delta p_{t}$ & & 75355 & 31813 & 18947 & 34827 \\
\hline Steam & 1.923 & 0.000015 & 0.078 & $\Delta p_{c}$ & 14228 & 200326 & 20299 & 14678 & 54714 \\
\hline & & & & $\Delta p_{r}$ & & 177 & 177 & 177 & 177 \\
\hline & & & & $\Delta p_{t}$ & & 200503 & 20476 & 14855 & 54891 \\
\hline & & & & Second ca & aign, rape & d oil & & & \\
\hline Crude oil & 882.1 & 0.0133 & 2.72 & $\Delta p_{c}$ & 154 & 83473 & 35650 & 21180 & 38780 \\
\hline & & & & $\Delta p_{r}$ & & 35 & 35 & 35 & 35 \\
\hline & & & & $\Delta p_{t}$ & & 83508 & 35685 & 21215 & 38816 \\
\hline Steam & 1.923 & 0.000015 & 0.078 & $\Delta p_{c}$ & 14265 & 209422 & 20450 & 14820 & 55012 \\
\hline & & & & $\Delta p_{r}$ & & $185^{1}$ & 185 & 185 & 185 \\
\hline & & & & $\Delta p_{t}$ & & 209607 & 20635 & 15005 & 55197 \\
\hline & & & & Third can & iign, sunflo & er oil & & & \\
\hline Crude oil & 882.3 & 0.0115 & 1.736 & $\Delta p_{c}$ & 113.4 & 35936 & 16906 & 9894 & 17273 \\
\hline & & & & $\Delta p_{r}$ & & 14 & 14 & 14 & 14 \\
\hline & & & & $\Delta p_{t}$ & & 35950 & 16910 & 9908 & 17287 \\
\hline Steam & 1.923 & 0.000015 & 0.050 & $\Delta p_{c}$ & 9104 & 89006 & 10390 & 7370 & 25613 \\
\hline & & & & $\Delta p_{r}$ & & 73 & 73 & 73 & 73 \\
\hline & & & & $\Delta p_{t}$ & & 89079 & 10443 & 7443 & 25686 \\
\hline Crude oil & 882.3 & 0.0115 & 2.049 & $\Delta p_{c}$ & 134 & 48566 & 21652 & 12778 & 22896 \\
\hline & & & & $\Delta p_{r}$ & & 20 & 20 & 20 & 20 \\
\hline & & & & $\Delta p_{t}$ & & 48586 & 21672 & 12798 & 22916 \\
\hline Steam & 1.923 & 0.000015 & 0.059 & $\Delta p_{c}$ & 10745 & 120291 & 13323 & 9519 & 33950 \\
\hline & & & & $\Delta p_{r}$ & & 101 & 101 & 101 & 101 \\
\hline & & & & $\Delta p_{t}$ & & 120392 & 13424 & 9620 & 34051 \\
\hline Crude oil & 882.3 & 0.0115 & 2.457 & $\Delta p_{c}$ & 161 & 67551 & 28406 & 16911 & 31177 \\
\hline & & & & $\Delta p_{r}$ & & 28 & 28 & 28 & 28 \\
\hline & & & & $\Delta p_{t}$ & & 67579 & 28434 & 16939 & 31205 \\
\hline Steam & 1.923 & 0.000015 & 0.071 & $\Delta p_{c}$ & 12885 & 167311 & 17495 & 12597 & 46230 \\
\hline & & & & $\Delta p_{r}$ & & 145 & 145 & 145 & 145 \\
\hline & & & & $\Delta p_{t}$ & & 167456 & 17640 & 12742 & 46375 \\
\hline Crude oil & 882.3 & 0.0115 & 2.713 & $\Delta p_{c}$ & 177 & 80881 & 32948 & 19705 & 36899 \\
\hline & & & & $\Delta p_{r}$ & & 35 & 35 & 35 & 35 \\
\hline & & & & $\Delta p_{t}$ & & 80816 & 32983 & 19740 & 36934 \\
\hline Steam & 1.923 & 0.000015 & 0.078 & $\Delta p_{c}$ & 14228 & 200326 & 20299 & 14678 & 54714 \\
\hline & & & & $\Delta p_{r}$ & & 177 & 177 & 177 & 177 \\
\hline & & & & $\Delta p_{t}$ & & 200503 & 20476 & 14855 & 54891 \\
\hline
\end{tabular}

The pressure drop in the crude oil circuit in PHE \#4 is smaller than in similar PHE \#2 because the route length is smaller and the section area is double in PHE \#4 comparing with PHE\#2. The same can be said about pressure drop on steam circuit. Also, the same considerations made at PHE \#2 about the differences between the results calculated with different correlations for the steam circuit are valid for the PHE\#4. 
A. A. Neagu et al. / Ovidius University Annals of Chemistry 27 (2016) 62-72

Table 5. Pressure drop values in PHE \#5 calculated with different correlations

\begin{tabular}{|c|c|c|c|c|c|c|c|c|c|}
\hline $\begin{array}{l}\text { Working } \\
\text { fluids }\end{array}$ & $\begin{array}{c}\text { Density, } \\
{\left[\mathrm{kg} / \mathrm{m}^{3}\right]}\end{array}$ & $\begin{array}{c}\text { Dinamic } \\
\text { viscosity, } \\
{[\mathrm{Pa} \cdot \mathrm{s}]}\end{array}$ & $\begin{array}{l}\text { Flow } \\
\text { rate, } \\
{[\mathrm{kg} / \mathrm{s}]}\end{array}$ & $\begin{array}{c}\text { Pressure } \\
\text { drop, }[\mathrm{Pa}]\end{array}$ & $\begin{array}{c}\text { Reynolds } \\
\text { number }\end{array}$ & $\begin{array}{c}\text { Kumar } \\
\text { correlation }\end{array}$ & $\begin{array}{c}\text { Bond I } \\
\text { correlation }\end{array}$ & $\begin{array}{c}\text { Buonopane } \\
\text { \& Troupe } \\
\text { correlation }\end{array}$ & $\begin{array}{c}\text { Muley } \\
\text { correlation }\end{array}$ \\
\hline \multicolumn{10}{|c|}{ First campaign, sunflower oil } \\
\hline \multirow{3}{*}{$\begin{array}{l}\text { Bleached } \\
\text { oil }\end{array}$} & \multirow[t]{3}{*}{895.2} & \multirow[t]{3}{*}{0.022} & \multirow[t]{3}{*}{1.940} & $\Delta p_{c}$ & \multirow[t]{3}{*}{25} & 26727 & 6321 & 8713 & 13034 \\
\hline & & & & $\Delta p_{r}$ & & 2 & 2 & 2 & 2 \\
\hline & & & & $\Delta p_{t}$ & & 26729 & 6323 & 8715 & 13036 \\
\hline \multirow[t]{3}{*}{ Water } & \multirow[t]{3}{*}{988.4} & \multirow[t]{3}{*}{0.00078} & \multirow[t]{3}{*}{2.546} & $\Delta p_{c}$ & \multirow[t]{3}{*}{921} & 11165 & 2240 & 4207 & 2556 \\
\hline & & & & $\Delta p_{r}$ & & 4 & 4 & 4 & 4 \\
\hline & & & & $\Delta p_{t}$ & & 11169 & 2244 & 4211 & 2560 \\
\hline \multirow{3}{*}{$\begin{array}{l}\text { Bleached } \\
\text { oil }\end{array}$} & \multirow[t]{3}{*}{895.2} & \multirow[t]{3}{*}{0.022} & \multirow[t]{3}{*}{2.193} & $\Delta p_{c}$ & 28 & 31757 & 7653 & 10718 & 15268 \\
\hline & & & & $\Delta p_{r}$ & & 3 & 3 & 3 & 3 \\
\hline & & & & $\Delta p_{t}$ & & 31760 & 7656 & 10721 & 15330 \\
\hline Water & 988.4 & 0.00078 & 2.878 & $\Delta p_{c}$ & 1038 & 13977 & 2778 & 5144 & 3287 \\
\hline & & & & $\Delta p_{r}$ & & 5 & 5 & 5 & 5 \\
\hline & & & & $\Delta p_{t}$ & & 13982 & 2783 & 5149 & 3292 \\
\hline Bleached & 895.2 & 0.022 & 2.490 & $\Delta p_{c}$ & 32 & 37996 & 9314 & 13315 & 18071 \\
\hline & & & & $\Delta p_{r}$ & & 4 & 4 & 4 & 4 \\
\hline & & & & $\Delta p_{t}$ & & 38000 & 9318 & 13319 & 18075 \\
\hline Water & 988.4 & 0.00078 & 3.268 & $\Delta p_{c}$ & 1179 & 17487 & 3480 & 6384 & 4065 \\
\hline & & & & $\Delta p_{r}$ & & 6 & 6 & 6 & 6 \\
\hline & & & & $\Delta p_{t}$ & & 17493 & 3486 & 6390 & 4158 \\
\hline Bleached & 895.2 & 0.022 & 2.755 & $\Delta p_{c}$ & 35 & 41614 & 10838 & 15808 & 20734 \\
\hline & & & & $\Delta p_{r}$ & & 5 & 5 & 5 & 5 \\
\hline & & & & $\Delta p_{t}$ & & 41619 & 10843 & 15813 & 20737 \\
\hline Water & 988.4 & 0.00078 & 3.616 & $\Delta p_{c}$ & 1305 & 21054 & 4163 & 7594 & 4905 \\
\hline & & & & $\Delta p_{r}$ & & 7 & 7 & 7 & 7 \\
\hline & & & & $\Delta p_{t}$ & & 21061 & 4170 & 7601 & 4912 \\
\hline & & & & Second ca & aign, rapes & d oil & & & \\
\hline Bleached & 893.6 & 0.018 & 2.76 & $\Delta p_{c}$ & 38 & 42012 & 10525 & 15455 & 19781 \\
\hline & & & & $\Delta p_{r}$ & & 5 & 5 & 5 & 5 \\
\hline & & & & $\Delta p_{t}$ & & 42017 & 10530 & 15460 & 19786 \\
\hline Water & 988.4 & 0.00078 & 3.622 & $\Delta p_{c}$ & 1306 & 21069 & 4173 & 7603 & 4930 \\
\hline & & & & $\Delta p_{r}$ & & 7 & 7 & 7 & 7 \\
\hline & & & & $\Delta p_{t}$ & & 21076 & 4180 & 7610 & 4937 \\
\hline & & & & Third can & aign, sunflo & r oil & & & \\
\hline Bleached & 894.65 & 0.0206 & 1.940 & $\Delta p_{c}$ & 30 & 23577 & 5739 & 8141 & 11227 \\
\hline & & & & $\Delta p_{r}$ & & 2 & 2 & 2 & 2 \\
\hline & & & & $\Delta p_{t}$ & & 23579 & 5741 & 8143 & 11229 \\
\hline Water & 988.4 & 0.00078 & 2.546 & $\Delta p_{c}$ & 919 & 11102 & 2223 & 4178 & 2556 \\
\hline & & & & $\Delta p_{r}$ & & 4 & 4 & 4 & 4 \\
\hline & & & & $\Delta p_{t}$ & & 11106 & 2227 & 4182 & 2560 \\
\hline Bleached & 894.65 & 0.0206 & 2.193 & $\Delta p_{c}$ & 34 & 28029 & 6935 & 10027 & 13243 \\
\hline oil & & & & $\Delta p_{r}$ & & 3 & 3 & 3 & 3 \\
\hline & & & & $\Delta p_{t}$ & & 28032 & 6937 & 10030 & 13246 \\
\hline Water & 988.4 & 0.00078 & 2.878 & $\Delta p_{c}$ & 1038 & 13872 & 2768 & 5146 & 3210 \\
\hline & & & & $\Delta p_{r}$ & & 5 & 5 & 5 & 5 \\
\hline & & & & $\Delta p_{t}$ & & 13877 & 2773 & 5151 & 3215 \\
\hline Bleached & 894.65 & 0.0206 & 2.490 & $\Delta p_{c}$ & 39 & 33529 & 8432 & 12442 & 15776 \\
\hline & & & & $\Delta p_{r}$ & & 4 & 4 & 4 & 4 \\
\hline & & & & $\Delta p_{t}$ & & 33533 & 8436 & 12446 & 15780 \\
\hline Water & 988.4 & 0.00078 & 3.268 & $\Delta p_{c}$ & 1179 & 17473 & 3474 & 6386 & 4065 \\
\hline & & & & $\Delta p_{r}$ & & 6 & 6 & 6 & 6 \\
\hline & & & & $\Delta p_{t}$ & & 17479 & 3480 & 6392 & 4071 \\
\hline Bleached & 894.65 & 0.0206 & 2.755 & $\Delta p_{c}$ & 43 & 38672 & 9854 & 14776 & 18181 \\
\hline oil & & & & $\Delta p_{r}$ & & 5 & 5 & 5 & 5 \\
\hline & & & & $\Delta p_{t}$ & & 38677 & 9859 & 14781 & 18186 \\
\hline Water & 988.4 & 0.00078 & 3.616 & $\Delta p_{c}$ & 1304 & 21003 & 4162 & 7584 & 4905 \\
\hline & & & & $\Delta p_{r}$ & & 7 & 7 & 7 & 7 \\
\hline & & & & $\Delta p_{t}$ & & 21010 & 4169 & 7591 & 4912 \\
\hline
\end{tabular}

The working fluids for gasket plate heat exchanger \#5 are bleached oil and water. The calculated pressure drop values for bleached oil and water are presented in Table 5. 
In this case too, the pressure drops calculated by Kumar relationship are higher than with other methods. The methods of Bond, Mulley and Buonopane give closer values.
In the heat exchanger \#6 the working fluids are deodorized oil - water. The pressure drop values for this equipment are presented in Table 6 .

Table 6. Pressure drop values in PHE \#6 calculated with different correlations

\begin{tabular}{|c|c|c|c|c|c|c|c|c|c|}
\hline $\begin{array}{l}\text { Working } \\
\text { fluids }\end{array}$ & $\begin{array}{l}\text { Density, } \\
{\left[\mathrm{kg} / \mathrm{m}^{3}\right]}\end{array}$ & $\begin{array}{c}\text { Dinamic } \\
\text { viscosity, } \\
{[\mathrm{Pa} \cdot \mathrm{s}]} \\
\end{array}$ & $\begin{array}{l}\text { Flow } \\
\text { rate, } \\
{[\mathrm{kg} / \mathrm{s}]}\end{array}$ & $\begin{array}{c}\text { Pressure } \\
\text { drop, } \\
{[\mathrm{Pa}]}\end{array}$ & $\begin{array}{l}\text { Reynolds } \\
\text { number }\end{array}$ & $\begin{array}{c}\text { Kumar } \\
\text { correlation }\end{array}$ & $\begin{array}{c}\text { Bond I } \\
\text { correlation }\end{array}$ & $\begin{array}{c}\text { Buonopane } \\
\text { \& Troupe } \\
\text { correlation }\end{array}$ & $\begin{array}{c}\text { Mulley } \\
\text { correlation }\end{array}$ \\
\hline \multicolumn{10}{|c|}{ First campaign, sunflower oil } \\
\hline \multirow{3}{*}{$\begin{array}{c}\text { Deodorized } \\
\text { oil }\end{array}$} & \multirow[t]{3}{*}{879.2} & \multirow[t]{3}{*}{0.0109} & \multirow[t]{3}{*}{1.736} & $\Delta p_{c}$ & \multirow[t]{3}{*}{19} & 4904 & 1123 & 1484 & 2500 \\
\hline & & & & $\Delta p_{r}$ & & 2 & 2 & 2 & 2 \\
\hline & & & & $\Delta p_{t}$ & & 4906 & 1125 & 1486 & 2502 \\
\hline \multirow[t]{3}{*}{ Water } & \multirow[t]{3}{*}{986.75} & \multirow[t]{3}{*}{0.00074} & \multirow[t]{3}{*}{6.02} & $\Delta p_{c}$ & \multirow[t]{3}{*}{979} & 10533 & 2099 & 3937 & 2437 \\
\hline & & & & $\Delta p_{r}$ & & 24 & 24 & 24 & 24 \\
\hline & & & & $\Delta p_{t}$ & & 10558 & 2124 & 3962 & 2457 \\
\hline \multirow{3}{*}{$\begin{array}{c}\text { Deodorized } \\
\text { oil }\end{array}$} & \multirow[t]{3}{*}{879.2} & \multirow[t]{3}{*}{0.0109} & \multirow[t]{3}{*}{2.049} & $\Delta p_{c}$ & 23 & 6137 & 1449 & 1963 & 3059 \\
\hline & & & & $\Delta p_{r}$ & & 3 & 3 & 3 & 3 \\
\hline & & & & $\Delta p_{t}$ & & 6140 & 1451 & 1966 & 3062 \\
\hline Water & 986.75 & 0.00074 & 7.100 & $\Delta p_{c}$ & 1154 & 14186 & 2827 & 5205 & 3316 \\
\hline & & & & $\Delta p_{r}$ & & 28 & 28 & 28 & 28 \\
\hline & & & & $\Delta p_{t}$ & & 14215 & 2855 & 5234 & 3345 \\
\hline Deodorized & 879.2 & 0.0109 & 2.475 & $\Delta p_{c}$ & 27 & 8003 & 1920 & 2678 & 3854 \\
\hline oil & & & & $\Delta p_{r}$ & & 4 & 4 & 4 & 4 \\
\hline & & & & $\Delta p_{t}$ & & 8007 & 1924 & 2682 & 3858 \\
\hline Water & 986.75 & 0.00074 & 8.500 & $\Delta p_{c}$ & 1379 & 19611 & 3706 & 7046 & 4646 \\
\hline & & & & $\Delta p_{r}$ & & 41 & 41 & 41 & 41 \\
\hline & & & & $\Delta p_{t}$ & & 19652 & 3747 & 7087 & 4688 \\
\hline Deodorized & 879.2 & 0.0109 & 2.713 & $\Delta p_{c}$ & 30 & 9205 & 2234 & 3171 & 4389 \\
\hline oil & & & & $\Delta p_{r}$ & & 5 & 5 & 5 & 5 \\
\hline & & & & $\Delta p_{t}$ & & 9210 & 2239 & 3176 & 4394 \\
\hline Water & 986.75 & 0.00074 & 9.400 & $\Delta p_{c}$ & 1528 & 23614 & 4656 & 8375 & 5584 \\
\hline & & & & $\Delta p_{r}$ & & 50 & 50 & 50 & 50 \\
\hline & & & & $\Delta p_{t}$ & & 23664 & 4706 & 8425 & 5634 \\
\hline & & & & Second & ampaign, $r$ & peseed oil & & & \\
\hline Deodorized & 878.7 & 0.012 & 2.720 & $\Delta p_{c}$ & 27 & 9449 & 2268 & 3167 & 4543 \\
\hline oil & & & & $\Delta p_{r}$ & & 5 & 5 & 5 & 5 \\
\hline & & & & $\Delta p_{t}$ & & 9454 & 2273 & 3172 & 4547 \\
\hline Water & 986.75 & 0.00074 & 9.436 & $\Delta p_{c}$ & 1533 & 23868 & 4708 & 8458 & 5612 \\
\hline & & & & $\Delta p_{r}$ & & 50 & 50 & 50 & 50 \\
\hline & & & & $\Delta p_{t}$ & & 23919 & 4758 & 8509 & 5663 \\
\hline & & & & Third c & mpaign, su & flower oil & & & \\
\hline Deodorized & 879.2 & 0.0116 & 1.736 & $\Delta p_{c}$ & 18 & 4915 & 1117 & 1461 & 2540 \\
\hline oil & & & & $\Delta p_{r}$ & & 2 & 2 & 2 & 2 \\
\hline & & & & $\Delta p_{t}$ & & 4917 & 1119 & 1463 & 2542 \\
\hline Water & 986.75 & 0.00074 & 6.022 & $\Delta p_{c}$ & 979 & 10568 & 2113 & 3947 & 2440 \\
\hline & & & & $\Delta p_{r}$ & & 20 & 20 & 20 & 20 \\
\hline & & & & $\Delta p_{t}$ & & 10589 & 2133 & 3968 & 2460 \\
\hline Deodorized & 879.2 & 0.0116 & 2.049 & $\Delta p_{c}$ & 21 & 6210 & 1442 & 1936 & $3098^{\circ}$ \\
\hline oil & & & & $\Delta p_{r}$ & & 3 & 3 & 3 & 3 \\
\hline & & & & $\Delta p_{t}$ & & 6213 & 1445 & 1939 & 3101 \\
\hline Water & 986.75 & 0.00074 & 7.108 & $\Delta p_{c}$ & 1156 & 14283 & 2841 & 5233 & 3320 \\
\hline & & & & $\Delta p_{r}$ & & 29 & 29 & 29 & 29 \\
\hline & & & & $\Delta p_{t}$ & & 14312 & 2870 & 5261 & 3349 \\
\hline Deodorized & 879.2 & 0.0116 & 2.457 & $\Delta p_{c}$ & 26 & 8024 & 1909 & 2637 & 3890 \\
\hline oil & & & & $\Delta p_{r}$ & & 4 & 4 & 4 & 4 \\
\hline & & & & $\Delta p_{t}$ & & 8028 & 1913 & 2641 & 3894 \\
\hline Water & 986.75 & 0.00074 & 8.523 & $\Delta p_{c}$ & 1386 & 19867 & 3930 & 7125 & 4652 \\
\hline & & & & $\Delta p_{r}$ & & 41 & 41 & 41 & 41 \\
\hline & & & & $\Delta p_{t}$ & & 19908 & 3972 & 7166 & 4693 \\
\hline Deodorized & 879.2 & 0.0116 & 2.713 & $\Delta p_{c}$ & 28 & 9226 & 2224 & 3120 & 4422 \\
\hline oil & & & & $\Delta p_{r}$ & & 5 & 5 & 5 & 5 \\
\hline & & & & $\Delta p_{t}$ & & 9231 & 2229 & 3125 & 4426 \\
\hline Water & 986.75 & 0.00074 & 9.410 & $\Delta p_{c}$ & 1530 & 23635 & 4691 & 8430 & 5590 \\
\hline & & & & $\Delta p_{r}$ & & 50 & 50 & 50 & 50 \\
\hline & & & & $\Delta p_{t}$ & & 23685 & 4741 & 8481 & 5641 \\
\hline
\end{tabular}


In the PHE \#6, the pressure drop calculated with Kumar correlation is higher than after the use of other methods, and all the other considerations for PHE\# 3 and \#5 (oil-water) are valid for PHE\#6.

To compare all the pressure drop values obtained by applying different methods, the results of calculations with Buonopane \& Troupe correlation were taken as a reference and relative errors to this were calculated.

The relative error is calculated with Eq. 12:

relative error $=\frac{\Delta p_{x}-\Delta p_{\text {Buonopane }}}{\Delta p_{\text {Buonopane }}} \times 100[\%]$

The comparison was made only for liquid as a working fluid, because the original correlations were worked out on experimental data on liquids. Results are presented in Table 7:

Table 7. Average relative errors of pressure drop values calculated with different methods compared with those obtained with Buonopane \& Troupe method, $\%$.

\begin{tabular}{|c|c|c|c|}
\hline PHE \# & $\begin{array}{c}\text { Kumar } \\
\text { method }\end{array}$ & $\begin{array}{c}\text { Mulley } \\
\text { method }\end{array}$ & $\begin{array}{c}\text { Bond I } \\
\text { method }\end{array}$ \\
\hline 1 & +136.5 & -2.0 & -41.7 \\
\hline 2 & +114.0 & -36.4 & -37.7 \\
\hline 3 & +183.8 & -29.4 & -35.9 \\
\hline 4 & +171.63 & -9.0 & -35.7 \\
\hline 5 & +176.32 & +16.3 & -38.2 \\
\hline 6 & +191.01 & +11.7 & -36.1 \\
\hline
\end{tabular}

The pressure drop values calculated with Mulley relationship and Buonopane \& Troupe correlation were close and also Bond's equation gave results close to the previous but systematically underestimated. Kumar correlation gave results far from all the others and its application will lead to oversize.

Following this comparative study we recommend the relationships of Mulley and that of Buonopane to be used for the estimation of pressure drop in gasket plate heat exchangers. The Kumar correlation should be applied with caution since it results in oversizing.

\section{Conclusions}

Different models developed by Kumar, Mulley, Bond and Buonopane \& Troupe were applied in industrial conditions on six PHEs in an industrial plant, in different size and working with different fluids (oils with changing properties, cooling water and condensing steam).

The pressure drop values calculated with Mulley relationship and Buonopane correlation were very close and also Bond's equation gave results close to the previous but slightly underestimated. Kumar correlation gave results far from all the others and its application will lead to oversize.
Following this comparative study we recommend the relationships of Mulley and Buonopane \& Troupe for the estimation of pressure drop in gasket plate heat exchangers. The Kumar correlation should be applied with caution since it results in oversizing.

These correlations wouldn't be considered for the calculation of pressure drop on condensing steam circuits since they weren't worked out for this type of fluid.

\section{References}

[1] C.S. Fernandes, R.P. Dias, J.M. Maia, Recent Patents on Mechanical Engineering 1 (3), 198205 (2008)

[2] A.H.K. Al-Tae, Comparative study of temperature control in a heat exchanger process. M. Sc. Thesis, Chemical Engineering/Unit Operation, University of Technology (2011)

[3] D.H. Han, K.J. Lee and Y.H. Kim, Journal of the Korean Physical Society 43 (1), 66-73, (2003)

[4] G.N. Jogi and M.S. Lawankar, International Journal of Emerging Technology and Advanced Engineering 2 (10), 110-115 (2012)

[5] I. Gherasim, M. Taws, N. Galanis and C.T. Nguyen, Applied Thermal Engineering 51, 346363 (2013)

[6] C.S. Fernandes, R.P. Dias, J.M. Noberga and J.M. Maia, Chemical Engineering and Processing 46, 825-833 (2007)

[7] Z.Luan, G. Zhang, M. Tian and M. Fan, Journal of Hydrodynamics 20(4), 524-529 (2008)

[8] G. Gulenoglu, F. Akturk, S. Aradag, N.S. Uzol, S. Kakac, International Journal of Thermal Sciences 75, 249-256, (2014)

[9] V.R. Naik, V.K. Matawala, International Journal of Engineering and Advanced Technology (IJEAT), 2 (4), 362-369 (2013)

[10] T. Kuppan, Heat Exchanger Design Handbook, Hemisphere Publishing Corporation, New York, (2000)

[11] S. Kakac, H. Liu, Heat Exchangers. Selection, Rating and Thermal Design, Second Edition, CRC Press (2002)

[12]F. Akturk, G. Gulben, S. Aradag, , N.S. Uzol, S. Kakac, $6^{\text {th }}$ International Advanced Technologies Symposion, 16-18 May,172-178 ( 2011)

[13] S. Kakac, H. Liu, Heat Exchangers Selection, Rating, and Thermal and Design, second ed., CRC Press, Boca Raton, 373-412 (2002)

[14]H. Kumar, The plate heat exchanger: construction and design, in: Proceedings First, UK National Conference on Heat Transfer, University of Leeds, Inst. Chem. Symp. Series No. 86, 1275-1288, (1984), cited in [6] 
[15] W.W. Focke, J. Zachariades, I. Olivier, The effect of the corrugation inclination angle on the thermo-hydraulic performance of plate heat exchangers, International Journal of Heat and Mass Transfer 28 (8), 1469-1479 (1985)

[16] A. Cooper, J.D. Usher, Heat Exchanger Design Handbook, Vol. 3, Section 3.7. Plate heat exchangers, Hemisphere, Washington, USA (1983)

[17]A.A. Neagu, I. Niţă, E. Botez, Ovidius University Annals of Chemistry 25 (2), 71-74 (2014)
[18] A.A Neagu, I. Niţă, E. Botez, S. Geacai, Ovidius University Annals of Chemistry 24 (2), 121-126 (2013)

[19]K. Raznjevic, Thermodynamic Tables and Diagrams (Tabele si diagrame termodinamice, in Romanian), Ed. Tehnica, Bucharest (1978)

Received: 10.04 .2016 Received in revised form: 08.06.2016

Accepted: 09.06.2016 